Authors post print version of:

Bull AMJ, Katchburian MV, Shih Y-F, Amis AA. Standardisation of the description of patellofemoral motion and comparison between techniques. Knee Surg. Sports Traumatol. Arthrosc 10, (2002), 184193.

The original publication is available at www.springerlink.com

\title{
Standardisation of the description of patellofemoral motion and comparison between different techniques
}

\author{
A.M.J. Bull, M.V. KATChBURIAN, Y-F. SHIH, A.A. AmIS
}

A.M.J. Bull ( (D) Department of Bioengineering, Imperial College, London SW7 2BX, United Kingdom

M.V. Katchburian: Orthopaedic Academic Unit, Rayne Institute, St. Thomas' Hospital, Lambeth Palace Road, London SE1 7EH, United Kingdom.

Y-F. Shih and A.A. Amis: Biomechanics Section, Mechanical Engineering Department, Imperial College, London SW7 2BX.

$$
\begin{aligned}
& \text { a.bull@ic.ac.uk } \\
& \text { +44207594 } 5186 \\
& \text { +448701254985 }
\end{aligned}
$$

This work was presented in part at the $12^{\text {th }}$ Conference of the European Society of Biomechanics, Dublin, August 2000. 


\section{ABSTRACT}

Patellofemoral motion is significant clinically, yet in the literature many different methods and terminologies are used, thus making comparison between papers difficult. The aim of this study was to review and explain the different methods used for the description of patellofemoral joint motion, compare these methods by experimentation, and propose a standardised method. We found three main methods for describing patellar motion: motion of the patella about femoral body fixed axes, about patellar body fixed axes, or a combination of these. Description about femoral body fixed axes does not make sense clinically. Description about patellar body fixed axes is straightforward, yet the definition of these axes is prone to error due to the lack of anatomical landmarks. The combination method makes most sense clinically and uses more easily found anatomical landmarks. Patellar flexion varied by up to $26 \%$ when describing the motion about different axes. Tilt and shift were highly sensitive to the choices of coordinate systems and the axes of motion. The pattern of rotation was consistent between all methods, however, differences between the methods increased with patellar flexion. We propose the description of patello-femoral motion in terms of shift (along a femoral medial-lateral axis), tilt (about the patellar long axis), rotation (about a floating patellar anterior-posterior axis) and flexion (about the femoral medial-lateral axis).

Keywords:

Patella, Patellofemoral joint, tracking, kinematics, motion description 


\section{INTRODUCTION}

Patellofemoral joint motion is of clinical interest, because of the large number of cases of patellofemoral disorders related to abnormal motion of the patella relative to the femur [8]. There are many papers in the literature that have attempted to describe patellar motion in a variety of situations, such as pre-and post-surgery, rehabilitation, in vivo, or in vitro, and preand post-total knee arthroplasty. Comparison between papers and validation of individual papers is difficult, because of the many different methods used to describe patellofemoral motion.

The aims of this paper are to review the different methods used for the description of patellofemoral joint motion, to compare these methods by experimentation, and propose a standardised technique. Others have analysed patellofemoral motion and sought to find a way of describing the motion that minimised inter specimen variation [3]. Although this paper is not addressing this important point, there may be scope for including the findings of Blankevoort and colleagues with the work presented here.

\section{METHODS USED TO DESCRIBE PATELLOFEMORAL JOINT MOTION}

The literature concerned with patellofemoral joint motion can be divided into two types: those that describe the motion of the patella relative to the femoral groove; and those that describe the motion of the patella relative to a fixed-body axis. The former are primarily imaging studies and will be dealt with briefly. This paper is more concerned with the latter, which can be described as six degree-of-freedom joint motion. Although this paper is concerned with patellofemoral motion, many of the papers reviewed have measured patellofemoral position at different points in time without reference to the dynamics of the 
joint. If the increments of position are small enough, then the description of motion may be acceptable. Patellar positions measured 'statically' may be different to those found 'dynamically', due to differences in muscle activity [4], and other factors such as direction of travel [14]. The exact rôle of speed of knee joint flexion-extension is undefined.

\subsection{Describing tracking in terms of the femoral groove}

Patellar motion and geometry have been studied using radiography, computerised tomography, static and dynamic MR imaging, and ultrasound in an attempt to find their relationship to anterior knee pain. Skyline radiographs or mid-patellar section by CT/MRI have been used to describe the patellar position relative to the femoral condyles or intercondylar groove, at different angles of knee flexion and with different loading conditions (partial weight-bearing or non-weight-bearing, with or without quadriceps contraction) [2]. A few studies have used ultrasound scanning to investigate malalignment of the patella $[13,20,28]$. Many parameters have been mentioned in these imaging studies, with the following most popular ones: sulcus angle, describing the geometry of the femoral articular surface; Merchant's congruence angle, a measure of lateral displacement and tilt of the patella; lateral patellofemoral angle and lateral patellar tilt angle denote the patellar tilt; both lateral patellar displacement and the bisect offset describe lateral patellar displacement [2] (Figure 1). The fact that most of these imaging studies were either done statically or without weight bearing is one of their disadvantages.

\section{Figure 1}

For the past ten years kinematic CT and MRI have been used to investigate the patellar motion during dynamic knee joint movement $[4,22,27]$. A significant drawback of the imaging studies has been the inability to describe the patellar motion in 3-dimensional space. All the parameters mentioned above describe two components of patellar motion: tilt, that is 
rotation about the supero-inferior axis of the patella, and lateral displacement, that is translation along the medio-lateral axis. Although CT, MRI, and ultrasound have the potential to show the 3-D relationship of the patellofemoral articulation, this has yet to be developed so that it can be used as a clinical tool. Sheehan et al. [26] showed that 3-D volume acquisition over the tracking cycle can be achieved by integrating velocity images. This promising technique requires 128-256 flexion/extension motion cycles that renders it incapable of being used clinically without further refinements and a significant reduction in the number of cycles required.

\subsection{Coordinate Systems}

Other quantitative techniques describe patellar motion along predefined axes and arbitrarily assign positive and negative values to specific directions of motion. The choice of axis can influence both the magnitude and perceived direction of motion and thus, the choice of axes is crucial. Comparison between existing reports in the literature is hindered by differences in these factors. Once the various techniques are clarified, a proposal for a standardised method can be made.

\section{PATELLOFEMORAL MOTION}

Patellofemoral motion is a full six degree-of-freedom (dof) motion. This means that the patella is not constrained in its motion, as is, for example, a fixed hinge joint. Therefore one may describe the motion of the patella as translations along and rotations about any axis and this can be related to the position of the femur. Of these six dof, four can be correlated with perceived clinically important motion variables: tilt, rotation, flexion, and shift. These are three rotations, and one translation. Anterior-posterior and proximal-distal translations are not clinically useful and these not usually referred to in the literature, although patellar position 
along these axes is significant, such as patella alta or infera. These clinical components of motion do not immediately relate to the position of the femur, they are concerned with the position of the patella in space. How the position of the patella in space relates to the position of the femur, or the local geometry of the trochlear groove, is a function of the definition of the description of patellofemoral motion, and relates directly to the clinical understanding of joint motion.

Three key variables must be accounted for to enable full understanding of the description of patellar motion:

1. In describing tilt, rotation, flexion and shift, what convention is used to describe the positive direction? This must correspond to clinical practice.

2. About and along which axes (patellar, femoral, or even tibial) do the rotations and translations occur?

3. What is the zero position of these motions?

In answering the above questions a few explanations of six degree of freedom motion need to made. Numerous techniques have been used to describe six-dof motion. The most commonly used methods in biomechanics are the use of the helical axis, and body-fixed axes [5]. Description of joint motion using the helical axis does not facilitate clinical description of motion, although it is elegant mathematically, whereas using body-fixed axes allows straightforward correlation between rotations about and translations along the body-fixed axes. These body-fixed axes are not necessarily mutually perpendicular (they are sometimes skewed), although to enable simple mathematics, they are most frequently described as mutually perpendicular [21]. Therefore, to describe patellar and femoral body-fixed axes, one only needs to describe the origin of the axis system, and the direction of two of the axes. The 
third axis is perpendicular to the other two. This is how the body-fixed axes are described below.

\subsection{Direction of patellofemoral motion}

In the literature, many techniques have been used to describe the direction of patellofemoral motion, the clearest of which is patellar flexion. Flexion of the patella is positive when the distal apex of the patella moves posteriorly relative to the proximal pole for angles of flexion of less than $90^{\circ}$. This is positive flexion (Figure 2a), following the flexion of the tibia.

\section{Figure 2}

Note that, although motion is different, in all three cases the patella can be said to have flexed. A more complete description can be given when the axes of motion have been described (this is discussed in the next section).

Patellar tilt is a rotation that occurs about an axis that is proximal-distal in the patella, and has been described in the literature as tilt (lateral or medial) or internal-external rotation $[17,32]$. Lateral tilt is equal and opposite to medial tilt. Tilt on its own is not descriptive and cannot be used uniquely without further explanation. Tilt is defined as being lateral when the lateral edge of the patella rotates towards the femur relative to the medial edge of the patella.

(Figure 2b). This corresponds to external rotation, as used by Lafortune [17], in that it follows the usual description of tibial rotation when the knee is extended.

Patellar rotation is normally reserved to describe a rotation about an axis perpendicular to the plane of the patella that is approximately coronal in extension, although this is dependent on the definition of the axes of motion, which is discussed below. This has been called abduction/adduction, twist, medial rotation, or lateral rotation in the literature. Lateral rotation is equal and opposite to medial rotation. Rotation is lateral when the patellar apex, 
i.e. the distal pole, moves laterally relative to the proximal pole (Figure 2c). This corresponds to abduction, as used by Lafortune [17], in that it follows the usual description of tibial abduction.

Patellar shift is the translation of the patella along a medial-lateral axis. Medial patellar shift is equal and opposite to lateral patellar shift. Patellar shift is lateral when the patella moves laterally relative to the femur as shown in Figure 2.

\subsection{Axes of motion}

It is apparent from the above that the description of the components of motion of the patella is missing the exact definitions of the axes along or about which the motions occur and the origin of the motion (that is, the zero position). In the literature, there are a number of significantly different ways in which these axes have been defined. These are discussed below and a critique is included. To begin, it is necessary to define the body-fixed axes of motion, and then define the axis about or along which the patellofemoral motion occurs. Finally, the origin of that motion needs to be described.

\subsubsection{Femoral Body-Fixed Axes}

Femoral body-fixed axes have been discussed widely in the literature with reference to tibiofemoral motion [5]. These can be mutually perpendicular axes, or skewed, as explained above. Typically these axes are described as either mechanical or anatomic [21]. The difference between these is that the long proximal-distal axis of the femur is either the 'mechanical axis', passing from the centre of the knee joint to the centre of the femoral head [11], or the 'anatomical axis' of the femur [17], which passes from the centre of the knee along the shaft of the femur (Figure 3). The mechanical axis is a functional axis as it is related to the function of the lower limb in gait. The anatomical axis is related neither to the function 
of the lower limb, nor the articular geometry of the patellofemoral joint. It is a description of the direction of the linear portion of the femoral shaft.

\section{Figure 3}

Perhaps a more relevant definition of the femoral long axis when describing patellar motion would be the direction of the femoral groove in the plane of either the mechanical or anatomical long axes, as shown in Figure 3. The definition of this axis is not consistent in the literature with values ranging from $15^{\circ}$ [30] to $2^{\circ}$ [18] from the mechanical axis. It is difficult to obtain this information as the groove is curved and slightly different definitions of this axis have been used. One of the reasons for this is that imaging data of the groove geometry from $\mathrm{X}$-rays is not consistent with the geometry of the articular cartilage [31]

The medial-lateral axis of the femur has been described in several ways: parallel to a line joining the medial and lateral epicondyles [21] or parallel to a line joining the posterior points of the femoral condyles $[11,17]$, or passing through the centres of the spherical posterior parts of the femoral condyles [16]. It has been shown that the choice of this axis will significantly affect the values obtained for certain components of patellar tracking [3]. The anteriorposterior axis of the femur is most often described as being mutually perpendicular to the other two axes. These definitions need to define both a direction, and a position. Not all these axis descriptions include a definition of the position of the axis relative to anatomical landmarks. They mostly use definitions of the direction of the axis and constrain this axis to pass through the 'centre of the knee', which can be described in many ways.

\subsubsection{Patellar Body-Fixed Axes}

Patellar body fixed axes have not been fully explored in the literature. Perhaps this is because anatomical landmarks defining the axes of motion are difficult to identify. One way that researchers have overcome this difficulty is by identifying the centre of the patella, and then 
aligning the axes of the patella with the femoral coordinate system in extension $[15,32]$. This doesn't allow patellar tracking pathologies near knee joint extension to be identified, because the assumption is made that the patellofemoral joint is in the correct alignment at extension. Also, this is fraught with experimental difficulties, because the loading at extension has a large effect on the position of the patella relative to the femur. This means that simply aligning the axes at extension also requires a full definition of the loading applied. This loading is hard to quantify in vivo, and hard to apply in vitro. Another technique has been used, in which the external anatomical landmarks on the patella are used. Various different techniques for doing this are described in the literature: Sakai et al. [24] digitised two points on a medial-lateral axis (not described in full), a third point on the proximal pole, and the centre of the patella. Several authors digitised the medial-lateral axis and proximal-distal axis by the most prominent proximal/medial/distal/lateral points on the patella $[7,9,23]$. Being points located through the skin, these have an indirect relationship to the articular geometry, which must be further quantified if contact conditions are of interest. Nagamine et al [19] used a mechanical device that was fixed parallel to the anterior surface of the patella. The centre of the patella was defined as half way between the most medial and lateral points of the patella. All motions were measured relative to these fixed points. Other techniques have used $\mathrm{x}$-ray anatomical landmarks, by taking sagittal and coronal images, and then the long and medial-lateral axes of the patella can be located relative to tantalum markers [33] or by deducing rotations from the position of the patellar apex on x-rays [29]. There are others in the literature who have not fully described the location of the patellar fixed-body axes, and therefore comparison with other data is difficult [1]. 


\subsubsection{Definition of motion relative to the body-fixed axes}

There are three main methods for describing patellar motion used in the literature. These are in terms of rotations about and translations along femoral body fixed axes, or patellar body fixed axes, or a combination of the two. These are described below.

\subsubsection{Patellar motion in terms of femoral body fixed axes}

In this technique, shift is defined as the movement of the patella (most frequently, the centre of the patella) along the femoral medial-lateral axis. Lateral rotation is defined as the rotation of the patella about the femoral anterior-posterior axis. This can cause some confusion, in two ways: first, because an isolated lateral rotation of the patella about this axis (which is shown in Figure 4) does produce a lateral movement of the patellar apex relative to the proximal pole (Figure 2), although this is an absolute medial movement of the proximal pole, where the patellar apex doesn't translate much at all. Second, because after the patella has flexed $90^{\circ}$, this lateral rotation actually is clinically described as tilt. Lateral tilt is the rotation of the

patella about the femoral long axis and, similar to rotation, when the patella has flexed $90^{\circ}$, a rotation about this femoral long axis is clinically described as rotation. Patellar flexion is the rotation of the patella about the femoral medial-lateral axis (Figure 4).

\section{Figure 4}

\subsubsection{Patellar motion in terms of patellar body fixed axes}

Shift is defined as the movement of the patella along the patellar medial-lateral axis. Rotation is defined as the rotation of the patella about the patellar anterior-posterior axis. Tilt is the rotation of the patella about the patellar long axis; patellar flexion is the rotation of the patella about the patellar medial-lateral axis (Figure 5). This definition is clearly unsatisfactory. For 
example, a tilted patella will move in a different direction when it shifts than if it were not tilted.

\section{Figure 5}

\subsubsection{Patellar motion in terms of femoral and patellar body fixed axes}

This method is a combination of the two techniques described above, and is the most commonly used method in the literature. There are a number of variations possible but only one will be presented here as the most commonly used example [12]. Shift has been defined as the movement of the patella parallel to (i.e. along) the femoral medial-lateral axis; rotation as the rotation of the patella about a floating axis parallel to the patellar anterior-posterior axis and perpendicular to both the femoral medial-lateral axis and the patellar long axis; tilt as the rotation of the patella about the patellar long axis and patellar flexion as the rotation of the patella about the femoral medial-lateral axis (Figure 6).

This method corresponds closely to the Grood and Suntay [11] method used for describing tibio-femoral motion where tibial abduction/adduction corresponds to patellar lateral/medial rotation, and tibial rotation corresponds to patellar tilt. This is essentially a three cylindric open chain representation of the patello-femoral joint [21].

\section{Figure 6}

\subsubsection{Origin of motion}

A discussion of the origin of motion (zero position) is included for completeness. This is a difficult concept to define or reproduce, because the zero positions are highly dependent on the experimental conditions, and on the articular and soft-tissue geometry. Many researchers use an arbitrary position at which to zero all rotations and translations. For example, the definition of axes employed by van Kampen et al [32] makes all rotations zero in extension, 
by aligning the femoral and patellar axes at this point. The lack of positive patellar location at this position would suggest that another zero position may be more reproducible. Imaging studies, however, do not impose these arbitrary constraints, and use anatomical geometrical measurements to obtain the position and orientation of the patella relative to the femoral groove. A further complication with this definition arises from the significant effect of muscle tension/relaxation on patellar position when the knee is extended. Perhaps the more comprehensive methods of imaging the joint combined with measuring and describing the motion according to a body-fixed axis technique will allow better comparison between papers, although further discussion is required.

In summary, most papers in the literature measure relative changes in position from an arbitrary origin. Either this origin and the loading conditions on the joint must be standardised, so that comparisons between papers may be made, or this origin can be related to the geometry of the patello-femoral joint or other anatomical features in the distal femur, in each case, so that individual results may be understood in geometrical terms.

\subsubsection{Discussion on Description of Motion}

The three methods for describing patellar motion give different values for the separate components of motion when describing the same motion. Also, within each method for describing patellar motion the variations in the definition of the axes of motion can also cause significant differences in patellar tracking data $[3,10]$. This means that comparison between papers that are measuring the same things, but describing them in different ways, is difficult. Furthermore, if using a femoral fixed axis system, the nature or definition of the patellar movements changes progressively as the knee flexes, when considering rotations about the a$\mathrm{p}$ and long axes, between patellar tilt and rotation; this is confusing when communicating 
with clinicians even if the method is a unique description of the motion. Therefore, a numerical comparison between the methods is made below, based on experimental data.

\section{NUMERICAL COMPARISON BETWEEN DIFFERENT METHODS USED TO DESCRIBE PATELLOFEMORAL MOTION}

Based on the analysis and review above an experiment was conducted to show how different definitions of the description of motion and the axis systems used would lead to different results for patellar rotations and translations in a typical knee, across a range of $0-90^{\circ} \mathrm{knee}$ joint flexion.

A single cadaveric knee was mounted in a patellar-tracking rig [25]. An electromagnetic device was used to measure the motion of the patella relative to the femur [6]. The following anatomical landmarks were digitised: medial and lateral femoral epicondyles, most posterior points on the medial and lateral femoral condyles, two points along the anatomical long axis of the femur, the 'centre of the knee' which was defined as the deepest point of the femoral patellar groove as it meets the intercondylar notch of the femur, and the deepest point of the femoral groove at its most proximal point [11]. The patellar points used were the most medial, lateral, proximal, and distal points on the patella.

From these points the following axes were defined:

Femur: $\quad$ Long axis: Anatomical, Groove

Medial/Lateral Axis: epicondylar axis, posterior femoral condylar axis

Patella: $\quad$ Long axis, and medial/lateral axis.

A normal tracking pattern was obtained by flexing and extending the knee joint with no constraints and with a single load of $20 \mathrm{~N}$ applied to the patella via the central quadriceps tendon to simulate the quadriceps mechanism. The resultant motion was measured and the 
description of motion computed using the combinations of femoral and patellar axes described above.

\subsection{Results}

All results are compared to the results obtained for the combination method using the femoral epicondylar axis and the anatomical long axis. Figure 7 displays the extreme variation achieved when describing the main components of motion using the different techniques.

Flexion: Patellar flexion varied by less than $1^{\circ}$ over the whole range when describing the motion about either of the femoral medial-lateral axes. When using the patellar medial-lateral axis the maximum error was at the highest angle of patellar flexion $\left(11.7^{\circ}\right)$.

Tilt: both the choice of coordinate systems and the axes of motion affected the values of tilt. These difference increased as the knee joint flexed. Over a range of $3.5^{\circ}$ tilt when using the combination method of describing the motion, a range $11.1^{\circ}$ tilt was described using a description of patellar tilt about the femoral long axis.

Shift: The definition of the femoral medial-lateral axis had a small effect on the values of patellar shift; however, the shift pattern was the same. These differences were less than $1 \mathrm{~mm}$ in a range of $2.2 \mathrm{~mm}$ of shift. When the patellar medial-lateral axis was used to describe shift, the pattern of shift was altered and the maximum differences in describing shift were $22 \mathrm{~mm}$.

Rotation: The pattern of rotation was consistent between all methods, however, with increasing patellar flexion, differences between the methods increased to $3.4^{\circ}$ at maximum flexion.

\section{Figure 7}




\subsection{Discussion on the numerical comparison}

These results show that a normal tracking pattern may be described in very different ways when different axes of motion and choice of coordinate systems are used. Quantitative comparisons cannot be given in general, because of the differences in geometry between specimens, which means that offsets between different axes of motion will occur. This means that a simple "conversion" factor cannot be used to transpose from one method to another. Therefore, we have included below a proposal for the future, to enable comparisons between different research outputs to be made.

\section{A PROPOSAL FOR THE FUTURE}

There are three areas that can introduce either confusion, or differences in quoted values for clinical motion with regard to any joint motion, and patello-femoral motion in particular. We include a proposal for the future so that work can be compared and understood without any ambiguity.

\subsection{Clinical motions}

We propose the description of patello-femoral motion in terms of shift, tilt, rotation and flexion, where the definitions of these are as above. The motions are shown in Figure 6 and can be summarised as:

- Shift is the medial or lateral movement of the centre of the patella along a mediallateral axis defined as either fixed to the femur or patella,

- Tilt is the angular position of the patella about its own long axis, where lateral tilt follows the sense of external tibial rotation, 
- Rotation is the angular position of the patella about an axis parallel to its own anterior-posterior axis, 'tethered' to the femoral medial-lateral axis and the patellar long axis as a three-cylindric open chain mechanism, where lateral rotation follows the sense of abduction in tibio-femoral motion,

- Flexion is the angular position of the patella about a medial-lateral axis defined as either fixed to the femur or patella.

We suggest that other terms, such as abduction (for rotation) and internal/external rotation (for tilt) are not used, because this causes confusion.

\subsection{Axes of motion}

Due to different measurement techniques, protocols, experimental limitations and aims of individual research programmes it is not possible for everyone to use the same definition of the axes of motion. However, we suggest that each paper describing patello-femoral joint motion defines the axes used and quantifies the relationship between their axes and one of the standard methods described here. These can be described as: a femoral system based on mechanical, anatomical, or femoral groove axes; a patellar system based on either the femoral system in extension, or anatomical landmarks. Knowing the axes of motion it is then necessary to also describe how the clinical motions (proposal included above) relate to these axis systems. Again, these can be described in three ways: patellar motion relative to the femoral body-fixed axes; patellar motion relative to the patellar body-fixed axes; patellar motion relative to a combination of the above. If the third technique is used, then a fuller explanation is needed, because different combinations are theoretically possible. For example, the three-cylindric open chain mechanism could consist of a floating axis that is connected to a patellar medial-lateral axis and a femoral long axis. 
Certain descriptions should not be used, in particular, describing patellar rotation and tilt relative to femoral axes. These cause confusion, because, although the description of motion will be unique, the way in which these terms relate to the clinical terms is ambiguous. At $90^{\circ}$ of patellar flexion the rotation of the patella about its long axis will correspond to a rotation about the femoral anterior-posterior axis. This would be described as patellar rotation, where in clinical use this is understood as patellar tilt. Similarly, at $90^{\circ}$ flexion a rotation of the patella about its anterior-posterior axis will correspond to a rotation about the femoral long axis. This would be described as patellar tilt, where in clinical use this is understood as patellar rotation (Figure 8)

\section{Figure 8}

\subsection{Origin of motion}

Because of the difficulty in obtaining a consistent origin of motion for patellar position, we do not suggest a standardised approach. We propose that the zero position must be defined clearly for each experiment, including the loading conditions on the joint, such as quadriceps loading/direction and angle of knee joint flexion, for example. The question of the zero position relates back to the function of the extensor mechanism. Therefore, a possibility is that the zero position should be related to the position of the femoral axis, or even to the position of the mechanical axis of the knee during gait. Another approach would be to describe the zero position as a function of the articular geometry of the patellofemoral joint. Because of the wide variability of gait patterns and articular geometry it would be difficult to define a useful standard for the origin of motion. This should be the subject for further work. 


\section{SUMMARY}

In this paper different methods used to describe patellofemoral joint motion have been reviewed and discussed. Terminology present in the literature was compared, and standardised terminology was proposed describing patellar motion in terms of shift, tilt, rotation and flexion. Differences in the description of patellar motion, that were caused by using different coordinate systems were quantified experimentally for a typical knee, and a proposal presented in which patellar motion is described about and along the femoral mediallateral axis, and about the patellar long axis, and a floating axis parallel to the patellar anterior-posterior axis and perpendicular to both other axes.

\section{ACKNOWLEDGEMENTS.}

The Arthritis Research Campaign, the Fielding Trust, the Special Trustees of St. Thomas' Hospital, and the Taiwanese government supported this work.

\section{References}

1. Ahmed AM, Shi S, Hyder A, Chan KH (1988) The effect of quadriceps tension characteristics on the patellar tracking pattern. Trans Orthop Res Soc 13:280-280

2. Beaconsfield T, Pintore E, Maffulli N, Petri J (1994) Radiological measurements in patellofemoral disorders. A review. Clin Orthop Rel Res 308:18-28

3. Blankevoort L, Kwak SD, Ahmad CS, Gardner TR, Grelsamer RP, Henry JH, Ateshian GA, Mow VC (1996) Effects of Global and Anatomic Coordinate Systems on Knee Joint Kinematics. Trans Eur Soc Biomech 10:260 
4. Brossmann J, Muhle C, Schroder C, Melchert UH, Bull CC, Spielmann RP, Heller M (1993) Patellar tracking patterns during active and passive knee extension: evaluation with motion-triggered cine MR imaging. Radiol 187:205-212

5. Bull AMJ, Amis AA (1998) Knee joint motion: description and measurement. Proc Inst Mech Engrs, Part H, J Eng Med 212:357-372

6. Bull AMJ, Berkshire FH, Amis AA (1998) Accuracy of an electromagnetic measurement device and application to the measurement and description of knee joint motion. Proc Inst Mech Engrs, Part H, J Eng Med 212:347-355

7. Bull AMJ, Katchburian MV, Amis AA (1999) Measuring patellar tracking in vivo. Inst Phys Eng Med June conference

8. Ficat RP, Hungerford DS (1977) Disorders of the Patellofemoral Joint. Williams \& Walkins, Baltimore.

9. Goh JC, Lee PY, Bose K (1995) A cadaver study of the function of the oblique part of vastus medialis. J Bone Jnt Surg 77-B:225-231

10. Grelsamer RP, Klein JR (1998) The biomechanics of the Patellofemoral Joint. J Orthop Sports Phys Ther 28:286-298

11. Grood ES, Suntay WJ (1983) A joint coordinate system for the clinical description of three- dimensional motions: application to the knee. Trans Am Soc Mech Eng, J Biomech Eng 105:136-144

12. Hefzy MS, Jackson WT, Saddemi SR, Hsieh YF (1992) Effects of tibial rotations on patellar tracking and patello- femoral contact areas. J Biomed Eng 14:329-343

13. Joshi RP, Heatley.F.W. (1998) Imaging patellofemoral joint using ultrasound: a preliminary report. A comparison between normal subjects and patients with patellar maltracking. Knee 5:129-135 
14. Katchburian MV, Bull AMJ, Heatley FW, Amis AA (2000) Patellar tracking in vivo quantifying the effect of tibial tubercle medialisation. Trans Eur Soc Biomech 12:11-11

15. Koh TJ, Grabiner MD, De Swart RJ (1992) In vivo tracking of the human patella. J Biomech 25:637-643

16. Kurosawa H, Walker PS, Abe S, Garg A, Hunter T (1985) Geometry and Motion of the Knee for Implant and Orthotic Design. J Biomech 18:487-499

17. Lafortune MA (1984) The use of intra-cortical pins to measure the motion of the knee joint during walking. PhD Thesis, Penn State University

18. Mori A (1992) [A histological study of the augmented reconstruction of the anterior cruciate ligament in rabbits]. Nippon Ika Daigaku Zasshi 59:176-185

19. Nagamine R, Otani T, White SE, McCarthy DS, Whiteside LA (1995) Patellar tracking measurement in the normal knee. J Orthop Res 13:115-122

20. Nietosvaara Y, Aalto KA (1993) Ultrasounographic evaluation of patellar tracking in children. Clin Orthop Rel Res 297:62-64

21. Pennock GR, Clark KJ (1990) An anatomy-based coordinate system for the description of the kinematic displacements in the human knee. J Biomech 23:1209-1218

22. Powers CM, Shellock FG, Pfaff M (1998) Quantification of patellar tracking using kinematic MRI. J Mag Res Imag 8:724-732

23. Reider B, Marshall JL, Ring B (1981) Patellar tracking. Clin Orthop Rel Res 157:143148

24. Sakai N, Luo ZP, Rand JA, An K-N (1996) Quadriceps forces and patellar motion in the anatomical model of the patellofemoral joint. Knee 3:1-7

25. Senavongse W, Bull AMJ, Amis AA (2000) Effects of pathological abnormalities on patellar tracking. Trans ESSKA 9:P168 
26. Sheehan FT, Zajac FE, Drace JE (1998) Using cine phase contrast magnetic resonance imaging to non-invasively study in vivo knee dynamics. J Biomech 31:21-26

27. Shellock FG, Mink JH, Deutsch AL, Foo TK, Sullenberger P (1993) Patellofemoral joint: identification of abnormalities with active-movement, "unloaded" versus "loaded" kinematic MR imaging techniques. Radiol 188:575-578

28. Shih Y-F, Bull AMJ, McGregor AH, Humphries K, Amis AA (2000) Ultrasound evaluation of patellar lateral tracking. Trans Eur Soc Biomech 12:155-155

29. Sikorski JM, Peters J, Watt I (1979) The importance of femoral rotation in chondromalacia patellae as shown by serial radiography. J Bone Jnt Surg 61-B:435-442

30. Siu D, Rudan J, Wevers HW, Griffiths P (1996) Femoral articular shape and geometry: a three-dimensional computerized analysis of the knee. J Arthrop 11:166-173

31. Stäubli H-U, Durrenmatt U, Porcellini B, Rauschning W (1999) Anatomy and surface geometry of the patellofemoral joint in the axial plane. J Bone Jnt Surg 81-B:452-458

32. van Kampen A, Huiskes R (1990) The three-dimensional tracking pattern of the human patella. J Orthop Res 8:372-382

33. Veress SA, Lippert FG, Hou MC, Takamoto T (1979) Patellar tracking patterns measurement by analytical x-ray photogrammetry. J Biomech 12:639-650 
Fig. 1 Description of patellar motion relative to the femoral groove. These descriptions are used primarily in imaging studies and relate the position of the patella to the articular geometry.

Fig. 2 Description of patellar motion. The solid lines represent the initial position of the patella, the dotted lines, the second position.

(A) Patellar flexion. In all these cases flexion is positive, because the distal apex of the patella has moved posteriorly relative to the proximal pole of the patella, even though the axis of rotation may not be at the centre of the patella.

(B) Patellar lateral tilt. Tilt is lateral when the lateral edge of the patella rotates towards the femur relative to the medial edge of the patella.

(C) Lateral patellar rotation. Rotation is lateral as shown, with the distal pole moving laterally with respect to the proximal pole.

(D) Lateral patellar shift. Shift is lateral as shown.

Fig. 3 Femoral mechanical, groove, and anatomical axes. The mechanical long axis runs from the origin of the coordinate system at the centre of the knee through the centre of the femoral head, while the anatomical long axis passes along the shaft of the femur. The groove axis is not clearly defined.

Fig. 4 Patellar motion in terms of femoral body fixed axes.

Fig. 5 Patellar motion in terms of patellar body fixed axes.

Fig. 6 Patellar motion in terms of a combination of femoral and patellar body fixed axes. This is a three-cylindric open chain representation of the patello-femoral joint with the joint translation and rotation axes coincident.

Fig. 7 Variation in the description of components of patellar motion compared to the 'ideal' combination method of describing patellofemoral motion, represented by solid lines.

(A) Patellar flexion/extension cycle

(B) Tilt.

(C) Shift.

(D) Rotation.

Fig. 8 Tilt and rotation of patella at $0^{\circ}$ and $90^{\circ}$ flexion displaying the ambiguity when using femoral axes. 


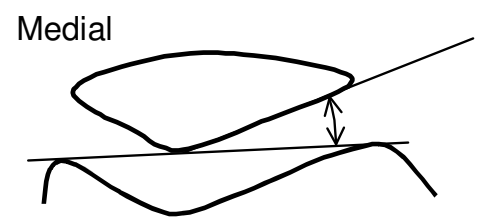

Lateral

patellofemoral angle

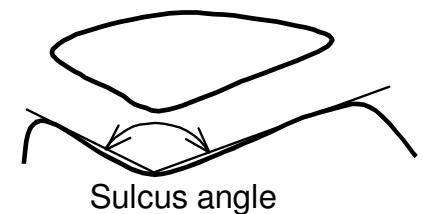

Sulcus angle

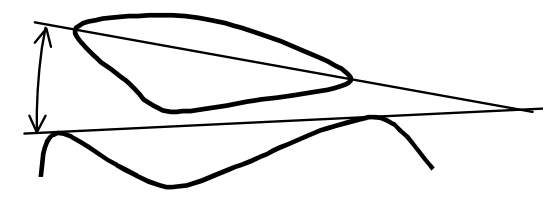

Lateral patellar tilt angle

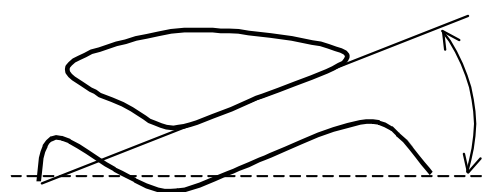

Patellar tilt angle (from the projected posterior condylar reference line)

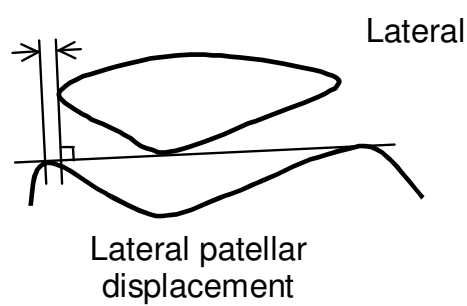

$(-) \leftrightarrow(+)$
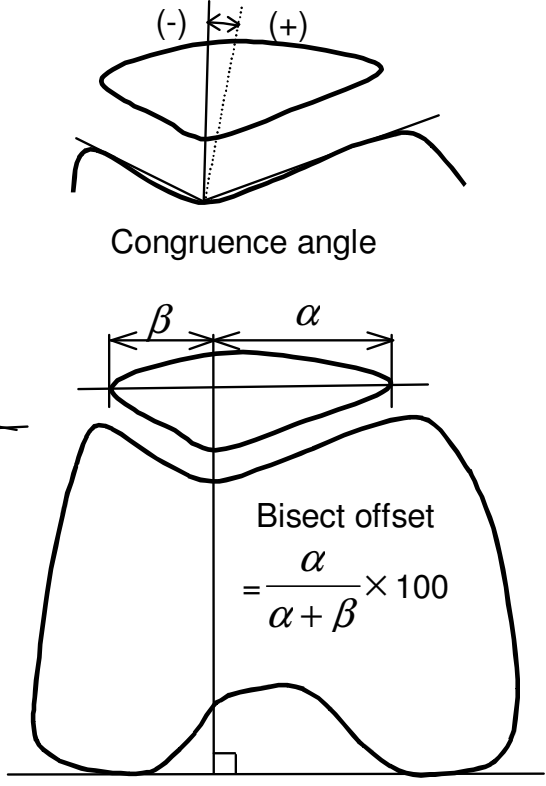

Bisect offset (\% of the patella width lateral to the line perpendicular to the posterior condylar reference line going through the deepest point of the trochlear groove) 


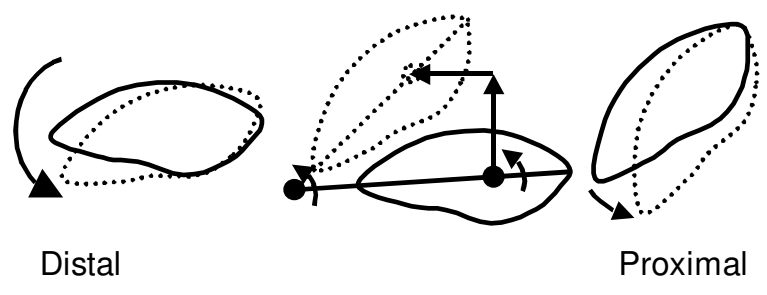

Flexion

(sagittal view)

(A)

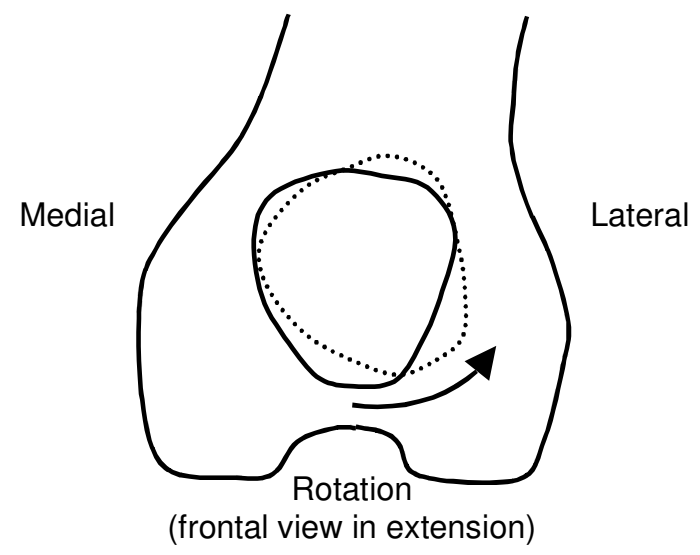

(C)
Medial Lateral

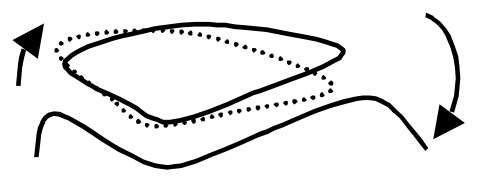

Tilt

(axial view)

(B)

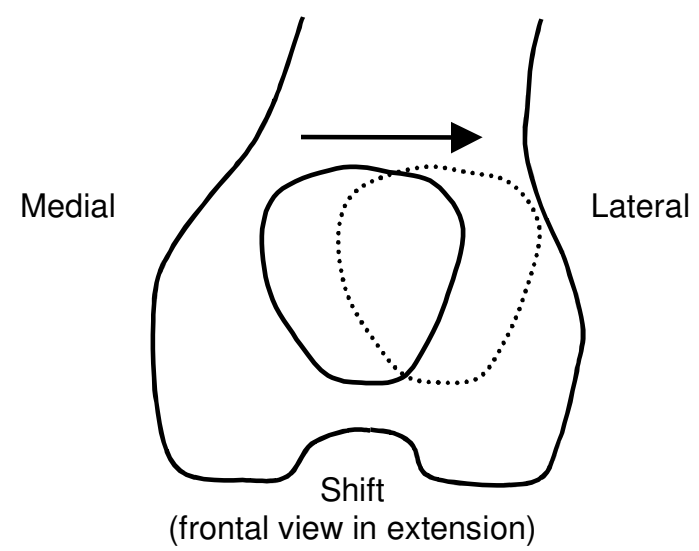

(D) 
Figure 3

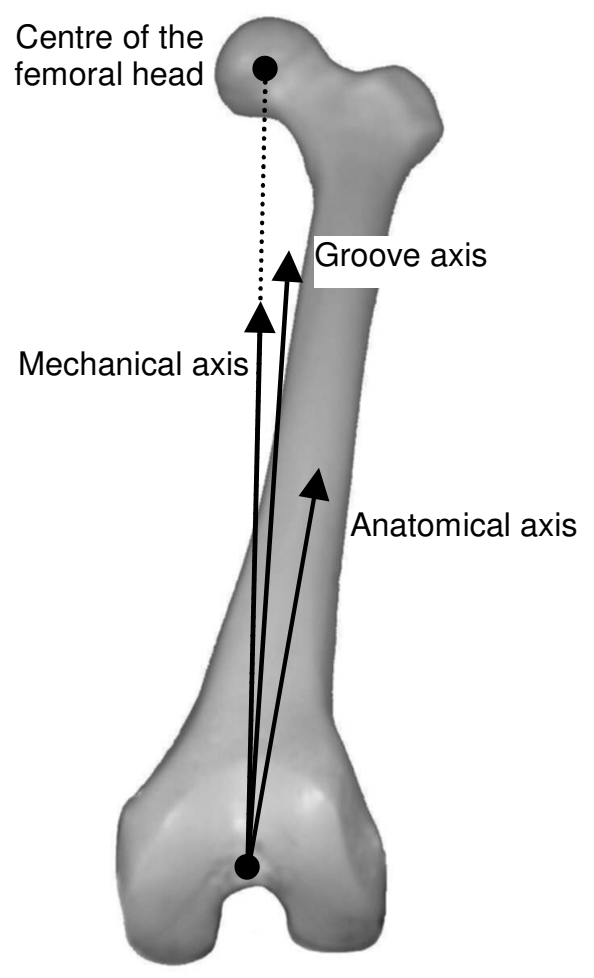




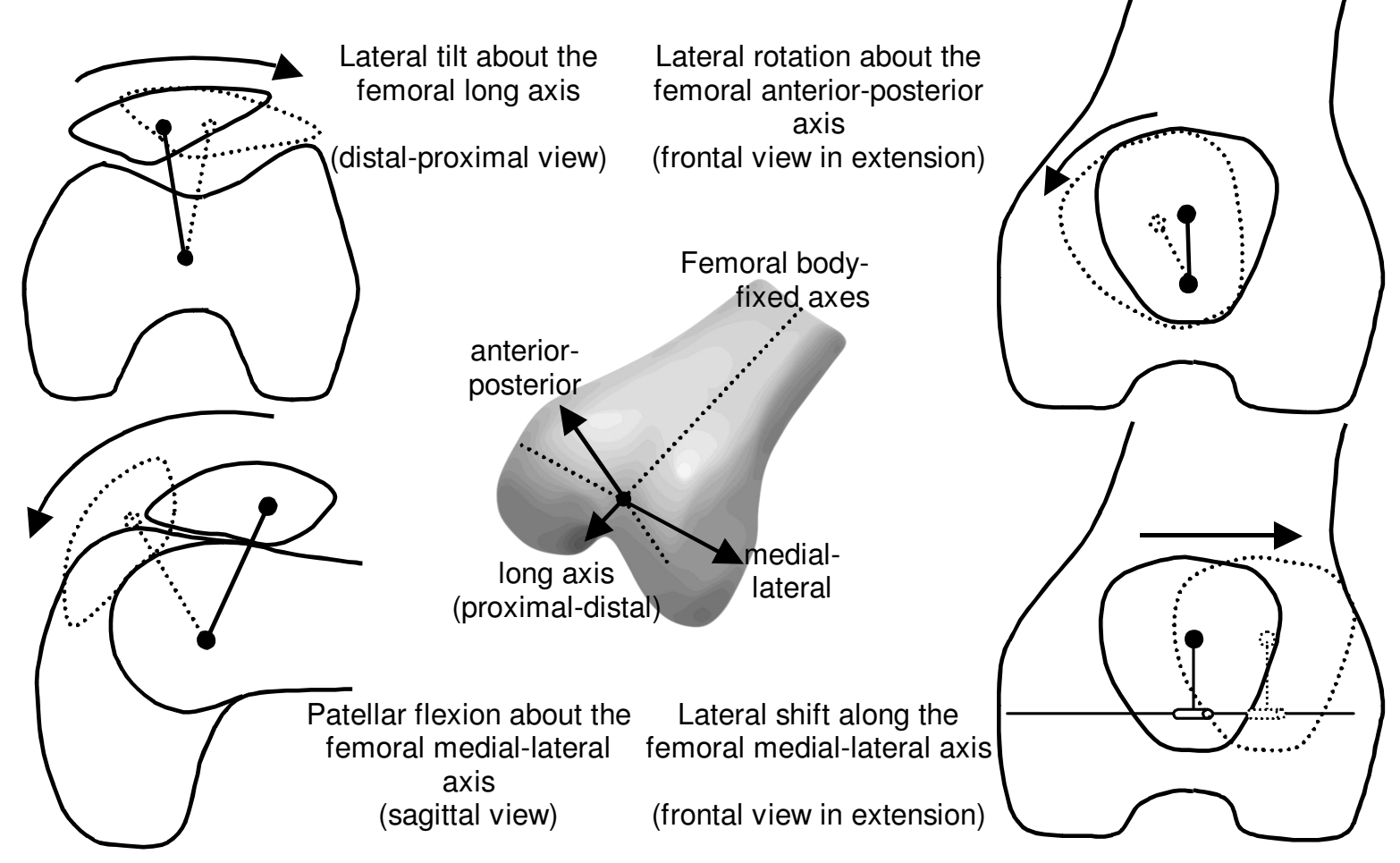




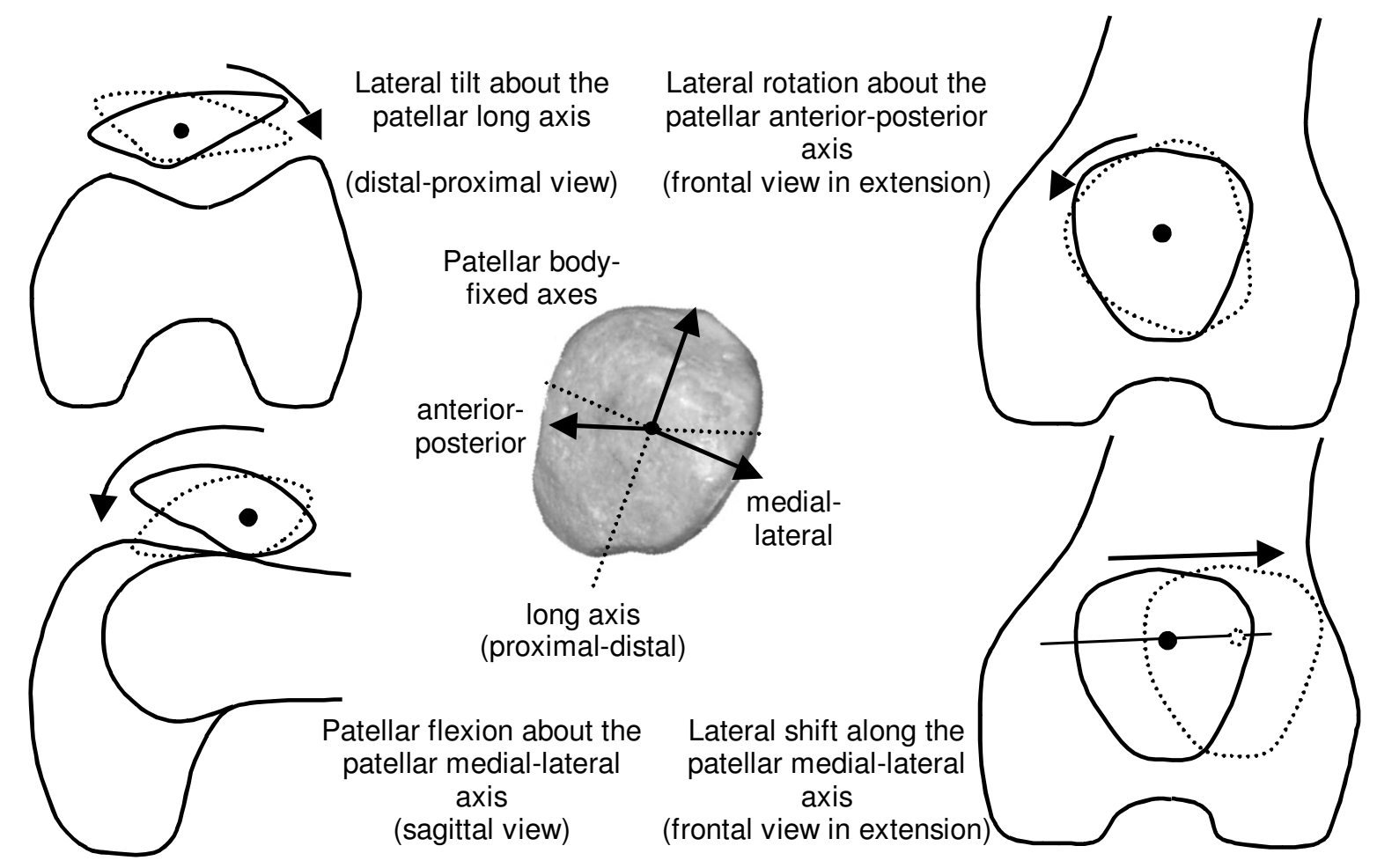




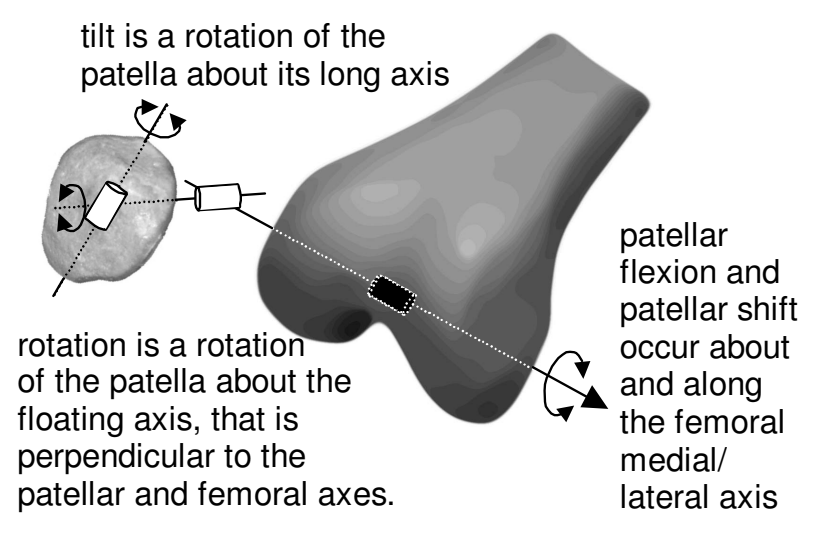



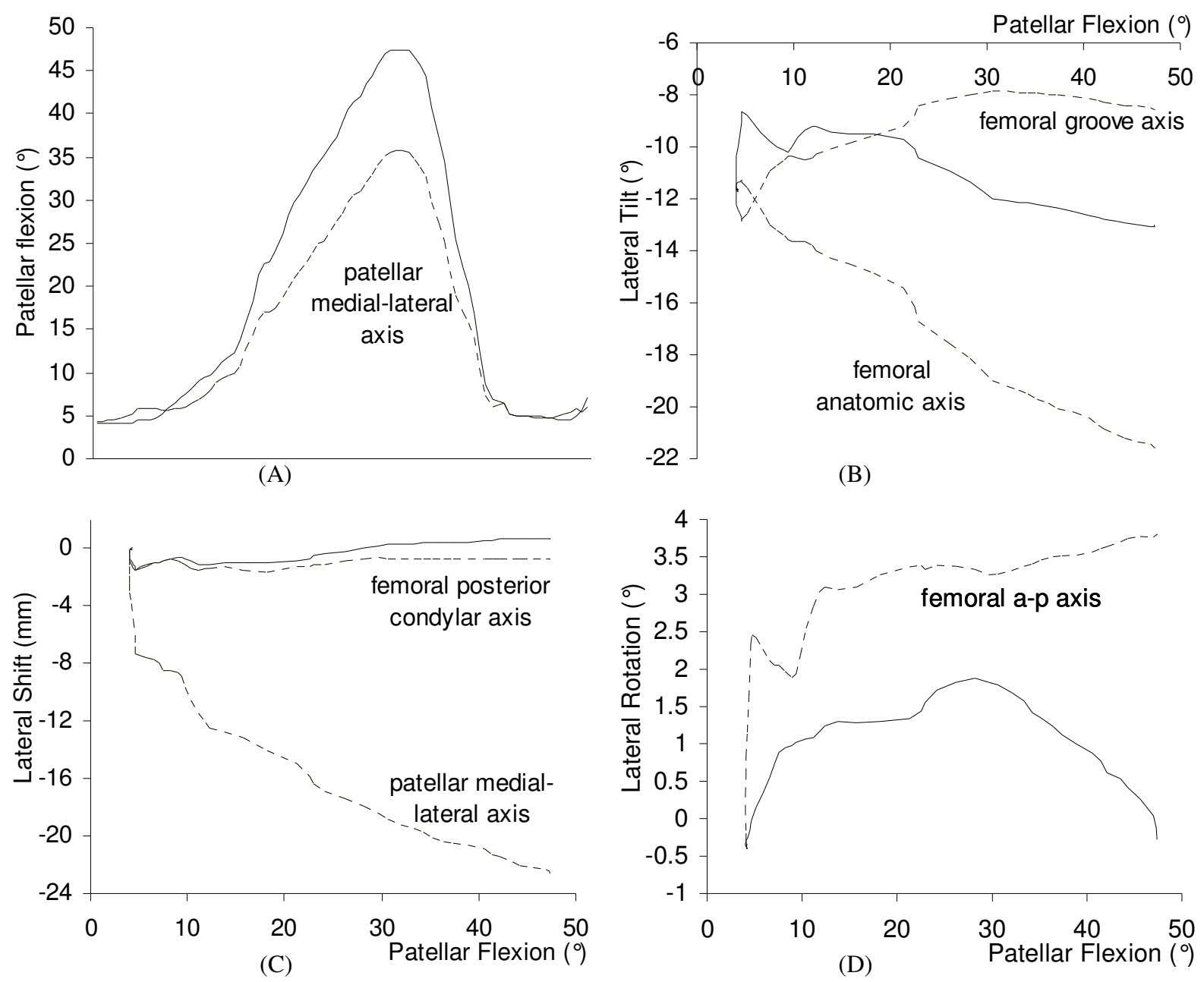


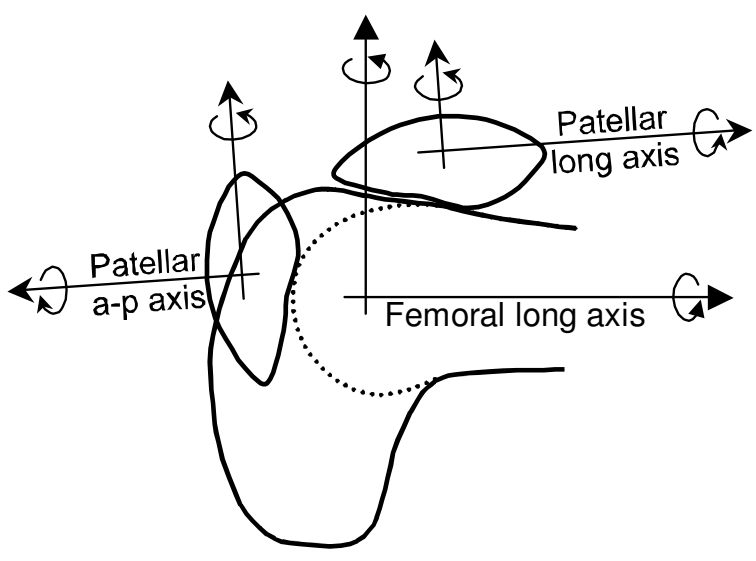

